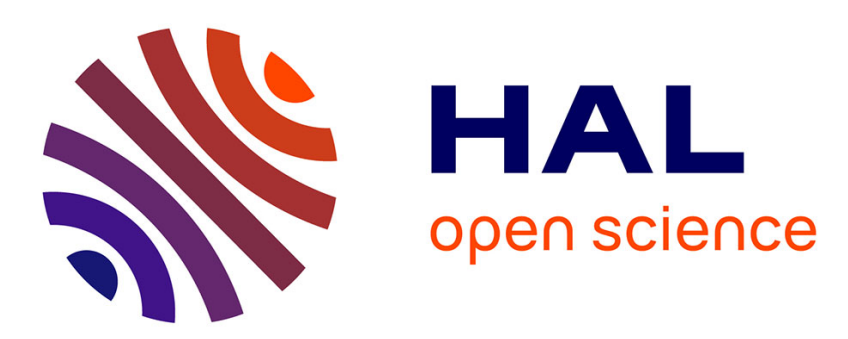

\title{
Modified marginal expected shortfall under asymptotic dependence
}

\author{
Juan-Juan Cai, Valérie Chavez-Demoulin, Armelle Guillou
}

\section{To cite this version:}

Juan-Juan Cai, Valérie Chavez-Demoulin, Armelle Guillou. Modified marginal expected shortfall under asymptotic dependence. Biometrika, 2017, 104, pp.243 - 249. 10.1093/biomet/asx005 . hal02393467

\section{HAL Id: hal-02393467 \\ https://hal.science/hal-02393467}

Submitted on 6 Dec 2019

HAL is a multi-disciplinary open access archive for the deposit and dissemination of scientific research documents, whether they are published or not. The documents may come from teaching and research institutions in France or abroad, or from public or private research centers.
L'archive ouverte pluridisciplinaire HAL, est destinée au dépôt et à la diffusion de documents scientifiques de niveau recherche, publiés ou non, émanant des établissements d'enseignement et de recherche français ou étrangers, des laboratoires publics ou privés. 


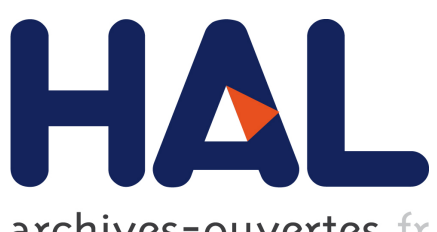

archives-ouvertes

\title{
Modified marginal expected shortfall under asymptotic dependence
}

\author{
Juan-Juan Cai, Valérie Chavez-Demoulin, Armelle Guillou
}

\section{To cite this version:}

Juan-Juan Cai, Valérie Chavez-Demoulin, Armelle Guillou. Modified marginal expected shortfall under asymptotic dependence. Biometrika, Oxford University Press (OUP), 2017, 104, pp.243 - 249. 10.1093/biomet/asx005 . hal-02393467

\section{HAL Id: hal-02393467 \\ https://hal.archives-ouvertes.fr/hal-02393467}

Submitted on 6 Dec 2019

HAL is a multi-disciplinary open access archive for the deposit and dissemination of scientific research documents, whether they are published or not. The documents may come from teaching and research institutions in France or abroad, or from public or private research centers.
L'archive ouverte pluridisciplinaire HAL, est destinée au dépôt et à la diffusion de documents scientifiques de niveau recherche, publiés ou non, émanant des établissements d'enseignement et de recherche français ou étrangers, des laboratoires publics ou privés. 


\title{
Modified marginal expected shortfall under asymptotic dependence
}

\author{
BY J.-J. CAI \\ Department of Applied Mathematics, Delft University of Technology, Mekelweg 4, 2628 CD, \\ Delft, The Netherlands \\ J.J.Cai@tudelft.nl \\ V. CHAVEZ-DEMOULIN \\ Faculty of Business and Economics, University of Lausanne, Bâtiment Anthropole, \\ 1015 Lausanne \\ Valerie.Chavez@unil.ch \\ AND A. GUILLOU \\ Institut de Recherche Mathématique Avancée, Université de Strasbourg, 7 rue René Descartes, \\ 67084 Strasbourg cedex, France \\ armelle.guillou@math.unistra.fr

\begin{abstract}
SUMMARY
We propose an estimator of the marginal expected shortfall by considering a log transformation of a variable which has an infinite expectation. We establish the asymptotic normality of our estimator under general assumptions. A simulation study suggests that the estimation procedure is robust with respect to the choice of tuning parameters. Our estimator has lower bias and mean squared error than the empirical estimator when the latter is applicable. We illustrate our methodology on a tsunami dataset.
\end{abstract}

Some key words: Asymptotic dependence; Asymptotic normality; Infinite mean model; Marginal expected shortfall; Tsunami data.

\section{INTRODUCTION}

Let $Z$ be a random variable belonging to the Fréchet domain of attraction with an extreme value index $\gamma$ $>0$, i.e.,

$$
\lim _{t \rightarrow \infty} \frac{\operatorname{pr}(Z>t x)}{\operatorname{pr}(Z>t)}=x^{-1 / \gamma}
$$

Statistical inference sometimes leads to an estimated extreme value index $\hat{\gamma} \geq 1$, giving a fitted model whose mean is infinite. This can arise in the context of operational risk (Chavez-Demoulin et al., 2016), of nuclear power accidents (Hofert \& Wuethrich, 2013; Wheatley et al., 2016) and of teletraffic data (Resnick, 1997), for instance. One way to approach this problem is to consider transformations of the form $W=Z^{a}$, with $a \gamma<1$, so that $W$ is in the Fréchet domain of attraction, i.e., its extreme value index is below 1. In this context, the method presented in Cai et al. (2015) can be used when one is interested in the conditional expectation $E\left\{W \mid Y>U_{Y}(1 / p)\right\}$, where $p$ is typically small and $Y$ is a random variable with tail quantile function $U_{Y}(t)=\inf \left\{y: F_{Y}(y) \geq 1-1 / t\right\}$, which is assumed to be asymptotically dependent on $W$. In practice the power transformation sometimes does not make sense or is difficult to interpret. In this paper, we consider the log transformation, which is commonly used in 
finance and for environmental scales such as Richter magnitudes. Define $X=\log Z$ with tail quantile function $U_{X}$. As shown by Proposition A1 in the Supplementary Material, $X$ now lies in the Gumbel domain of attraction, i.e.,

$$
\lim _{t \rightarrow \infty} \frac{U_{X}(t x)-U_{X}(t)}{\gamma}=\log x
$$

On the basis of a random sample of size $n,\left\{\left(Z_{1}, Y_{1}\right), \ldots,\left(Z_{n}, Y_{n}\right)\right\}$, our aim in this paper is to estimate

$$
\theta_{p}=E\left\{\log Z \mid Y>U_{Y}\left(\frac{1}{p}\right)\right\}
$$

where $p=p_{n} \rightarrow 0$ as $n \rightarrow \infty$. This quantity is known in the literature as the marginal expected shortfall; see, for instance, Caporin \& Santucci de Magistris (2012). The Supplementary Material contains the proofs of the main results together with some additional simulations and a second application.

\section{ESTIMATOR AND ASYMPTOTIC PROPERTIES}

To construct an estimator of $\theta_{p}$, we need some conditions on the dependence structure of the pair $(Z, Y)$ and on their marginal distributions. Often, we expect to see simultaneously high values of $Z$ and $Y$, so a particular assumption must be imposed on the upper-tail dependence of $(Z, Y)$. More precisely, we assume that the following limit exists for all $(x, y) \in[0, \infty]^{2} \backslash\{(\infty, \infty)\}$ :

$$
R_{t}(x, y)=t \operatorname{pr}\left\{1-F_{Z}(Z) \leq \frac{x}{t}, 1-F_{Y}(Y) \leq \frac{y}{t}\right\} \rightarrow R(x, y), \quad t \rightarrow \infty,
$$

where $F_{Z}$ and $F_{Y}$ denote the continuous marginal distribution functions of $Z$ and $Y$.

Concerning the marginal distributions, we assume that $Z$ is in the Fréchet domain of attraction, i.e., its 50 distribution satisfies (1), but we impose no conditions on the marginal distribution of $Y$ except continuity; therefore, applying any continuous increasing transformation to $Y$ will not change the marginal expected shortfall. Since we take the $\log$ transformation on $Z, X=\log Z$ is in the Gumbel domain of attraction and the copula of $(X, Y)$ is the same as that of $(Z, Y)$; hence the tail dependence structure of $(X, Y)$ also remains the same. Our results are valid for any positive $\gamma$, although our primary concern is the case $\gamma \geq 1$.

Throughout the paper, we assume positive $X$. From a practical point of view, the data are generally positive or can always be made positive; in the risk management context, for instance, one typically considers negative returns so that losses are on the positive side of the profit-and-loss distribution. However, values of $\mathrm{Z}$ may sometimes be below 1 , leading to negative $X$. This is the case for the tsunami data in Section 4 , where $Z$ corresponds to the water height in meters. One way to avoid negative $X$ is by changing units of $Z$, for example by converting the water height to centimeters before taking the logarithm. Theoretically, if $Z>0$ is in the maximum domain of attraction of a Fréchet distribution with an extreme value index $\gamma$, then this is also the case of the transformed variable $W=a Z$ for any $a>0$ with the same $\gamma$.

All the results in this paper will be derived under the second-order condition:

Condition 1. there exist $\beta>0$ and $\tau<0$ such that, as $t \rightarrow \infty$,

$$
\sup _{0<x \leq \infty, 1 / 2 \leq y \leq 2} \frac{\left|R_{t}(x, y)-R(x, y)\right|}{x^{\beta} \wedge 1}=O\left(t^{\tau}\right) .
$$

This condition is very general and is satisfied for instance by the two distributions considered in Section 3. It is the same condition as that of Cai et al. (2015), but with no constraint on $\beta$ other than positivity, and allows us to specify the rate of convergence in (3). The uniformity requirement in Condition 1 implies that if $t$ is large enough, $R(t, 1)$ is close to 1 , so it excludes asymptotic independence. Also, the fact that $y$ lies in $[1 / 2,2]$ is only a technical requirement for the proof and can be replaced by any interval of the form $[1-c, 1+d]$, for positive $c$ and $d$.

THEOREM 1. Under Condition 1 and (1), we have $\theta_{p} / U_{X}(1 / p) \longrightarrow 1$ as $p \rightarrow 0$. 
In view of Theorem 1, the first idea for estimating $\theta_{p}$ is to use an extreme quantile estimator of $U_{X}(1 / p)$. Let $X_{i}=\log Z_{i}(i=1, \ldots, n)$. We propose to use the maximum likelihood estimator,

$$
\hat{U}_{X}\left(\frac{1}{p}\right)=X_{n-k_{1}, n}+\hat{\gamma}\left(k_{1}\right) \log \frac{k_{1}}{n p},
$$

where $\hat{\gamma}\left(k_{1}\right)=k_{1}^{-1} \sum_{i=1}^{k_{1}}\left(X_{n-i+1, n}-X_{n-k_{1}, n}\right)$, with $X_{n-i+1, n}$ the $i$ th largest observation among the $X$-sample and $k_{1}$ an intermediate sequence, i.e., a sequence satisfying $k_{1} \rightarrow \infty$ and $k_{1} / n \rightarrow 0$ as $n \rightarrow \infty$. However, one cannot get the desired asymptotic normality using $\hat{U}_{X}(1 / p)$ as an estimator of $\theta_{p}$, because the convergence rate of $\hat{U}_{X}(1 / p)$ to its true value is $k_{1}^{1 / 2} / \log \left\{k_{1} /(n p)\right\}$, whereas the convergence rate in Theorem 1 is typically only of the order $\log (1 / p)$. To solve this, we must estimate $\theta_{p}$ more precisely. To understand the construction of our estimator heuristically, define $s_{1 / p}(x)=\left[1-F_{X}\left\{x U_{X}(1 / p)\right\}\right] / p$ and write

$$
\begin{aligned}
\theta_{p}= & U_{X}\left(\frac{1}{p}\right)+U_{X}\left(\frac{1}{p}\right) \int_{0}^{a_{n}}\left[R_{1 / p}\left\{s_{1 / p}(x), 1\right\}-1\right] d x \\
& \underbrace{-U_{X}\left(\frac{1}{p}\right) \int_{a_{n}}^{1}\left[1-R_{1 / p}\left\{s_{1 / p}(x), 1\right\}\right] d x}_{\Theta_{1}}+\underbrace{U_{X}\left(\frac{1}{p}\right) \int_{1}^{\infty} R_{1 / p}\left\{s_{1 / p}(x), 1\right\} d x}_{\Theta_{2}},
\end{aligned}
$$

where $a_{n}$ is a sequence such that $a_{n} \in(0,1)$ and $a_{n} \rightarrow 1$ as $n \rightarrow \infty$. The first term in (5) can be estimated by (4). By a suitable choice of $a_{n}$, the second term in (5) can be made negligible. Hence the task consists of estimating $\Theta_{1}$ and $\Theta_{2}$. Using a non-parametric estimator of $R(x, 1)$, we can justify the approximations

$$
\begin{aligned}
& \Theta_{1} \approx-\frac{1}{k_{2}} \sum_{i=1}^{n} \mathbb{1}_{\left(R_{i}^{X}<n-k_{2}+1, R_{i}^{Y} \geq n-k_{2}+1\right)}\left[\left\{\left(1-a_{n}\right) U_{X}\left(\frac{1}{p}\right)\right\} \wedge\left\{\gamma \log \left(\frac{n-R_{i}^{X}}{k_{2}}\right)\right\}\right], \\
& \Theta_{2} \approx \frac{\gamma}{k_{1}} \sum_{i=1}^{n} \mathbb{1}_{\left(R_{i}^{X}>n-k_{1}+1, R_{i}^{Y}>n-k_{1}+1\right)} \log \left(\frac{k_{1}}{n-R_{i}^{X}+1}\right),
\end{aligned}
$$

where $R_{i}^{X}$ and $R_{i}^{Y}$ denote the ranks of $X_{i}$ and $Y_{i}$ in their respective samples, and $k_{2}$ is an intermediate sequence, i.e., $k_{2} \rightarrow \infty$ and $k_{2} / n \rightarrow 0$ as $n \rightarrow \infty$, possibly different from $k_{1}$. Plugging $\hat{U}_{X}(1 / p)$ and $\hat{\gamma}\left(k_{1}\right)$ into (6) and (7), we obtain our final estimator,

$$
\begin{aligned}
\hat{\theta}_{p}= & \hat{U}_{X}\left(\frac{1}{p}\right)-\frac{1}{k_{2}} \sum_{i=1}^{n} \mathbb{1}_{\left(R_{i}^{X}<n-k_{2}+1, R_{i}^{Y} \geq n-k_{2}+1\right)}\left[\left\{\left(1-a_{n}\right) \hat{U}_{X}\left(\frac{1}{p}\right)\right\} \wedge\left\{\hat{\gamma}\left(k_{1}\right) \log \left(\frac{n-R_{i}^{X}}{k_{2}}\right)\right\}\right] \\
& +\frac{\hat{\gamma}\left(k_{1}\right)}{k_{1}} \sum_{i=1}^{n} \mathbb{1}_{\left(R_{i}^{X}>n-k_{1}+1, R_{i}^{Y}>n-k_{1}+1\right)} \log \left(\frac{k_{1}}{n-R_{i}^{X}+1}\right),
\end{aligned}
$$

where $\mathbb{1}_{A}$ denotes the indicator function of a subset $A$ and $\wedge$ the minimum function. For asymptotic normality of $\hat{\theta}_{p}$, we also need a second-order condition on the marginal distribution of $Z$ or equivalently on $X$. Specifically, we assume that $Z$ has a Hall-type distribution, meaning that, with $C>0, D \in \mathbb{R}$, $\rho<0$,

$$
1-F_{Z}(t)=C t^{-1 / \gamma}\left[1+D t^{\rho / \gamma}\{1+o(1)\}\right]=t^{-1 / \gamma} \ell_{Z}(t), \quad t \rightarrow \infty
$$

where the function $\ell_{Z}$ is slowly varying at infinity; see Hall (1982). Model (8) is satisfied by most distributions in the Fréchet domain of attraction; see for instance, Table 2.1 in Beirlant et al. (2004).

Theorem 2. Assume Condition 1 and the Hall model (8) with $\gamma>0$. Suppose also that (3) holds and that the partial derivatives $\partial R(x, y) / \partial x$ and $\partial R(x, y) / \partial y$ are continuous. Let $a_{n} \in(0,1)$ be a sequence tending to one such that $s_{1 / p}\left(a_{n}\right) \leq\left(n / k_{2}\right)\{n /(n+1)\}$. Consider two intermediate sequences $k_{1}$ and $k_{2}$ such that $k_{1}=o\left(k_{2}\right), n p=o\left(k_{1}\right), \log (n p)=o\left(k_{1}^{1 / 2}\right), k_{1}=o\left\{n^{2 \tau /(2 \tau-1)}\right\}, k_{1}=o\left\{n^{2 \rho /(2 \rho-1)}\right\}$, 


$$
\begin{aligned}
& U_{X}(1 / p)\left(1-a_{n}\right) / \log \left\{k_{1} /(n p)\right\} \rightarrow 0, k_{1}^{1 / 2} U_{X}(1 / p)\left(1-a_{n}\right) / \log \left\{k_{1} /(n p)\right\} \rightarrow \infty \text { and } \\
& \quad \frac{k_{1}^{1 / 2} U_{X}(1 / p)}{\log \left\{k_{1} /(n p)\right\}} \max \left[p^{\tau\left(a_{n}-1\right)},\left(\frac{n}{k_{2}}\right)^{\tau}\left(1-a_{n}\right), \frac{n^{1 / 2}}{k_{2}}\left(1-a_{n}\right), \frac{\left\{k_{2} /(n p)\right\}^{\rho}}{U_{X}(1 / p)}\right] \rightarrow 0 .
\end{aligned}
$$

Then $k_{1}^{1 / 2}\left(\hat{\theta}_{p}-\theta_{p}\right) / \log \left\{k_{1} /(n p)\right\}$ converges in distribution to a centered normal distribution with variance $\gamma^{2}$.

Remark 1 . In practice, the value of $p$ is typically determined by the application. Thus, when applying the estimation method, one only needs to choose $k_{1}, k_{2}$ and $a_{n}$. Below, we explain the implication of our conditions for these three sequences from a practical point of view. First of all, under the Hall model (8), we have $U_{X}(1 / p)=-\gamma \log p\{1+o(1)\}$. Hence the factor $U_{X}(1 / p) / \log \left\{k_{1} /(n p)\right\}$, which appears several times in the conditions of Theorem 2, does not play a role, compared to other terms when we assume, as usual, that $k_{1}=\left\lfloor n^{a}\right\rfloor$ and $k_{2}=\left\lfloor n^{b}\right\rfloor$ for positive $a$ and $b$, where $\lfloor\cdot\rfloor$ denotes the integer part. The conditions for the sequences in Theorem 2 can then be implied by

$$
\begin{aligned}
& a \in\left(0, \min \left\{\frac{-2 \tau}{1-2 \tau}, \frac{-2 \rho}{1-2 \rho} b,-2 \tau(1-b), 2 b-1\right\}\right), \\
& n p=o\left(k_{1}\right), \quad \log (n p)=o\left(k_{1}^{1 / 2}\right), \\
& p^{a_{n}-1}=O\left(n^{1-b}\right), \quad n^{a / 2} p^{\tau\left(a_{n}-1\right)}=o(1), \\
& a_{n} \rightarrow 1, \quad k_{1}^{1 / 2}\left(1-a_{n}\right) \rightarrow \infty .
\end{aligned}
$$

The first condition in (9) is similar to Condition (d) in Cai et al. (2015). The second line of (9) represents the usual constraints on $p$ due to the asymptotic normality of the quantile estimator, see Proposition A2 in the Supplementary Material. The third line of (9) links $a$ and $b$ to $p$ and $a_{n}$ and the last implies that $a_{n}$ should tend to unity, but not too quickly. Our simulation study suggests that our estimator is insensitive to the choice of $a_{n}$.

\section{Simulation StUdy}

To assess the performance of our estimator in practice, we simulated $B=500$ samples from the following two examples.

Example 1. The absolute value of a standard bivariate Cauchy distribution.

Example 2. The absolute value of a standard bivariate Student(1-1) distribution, with the first component raised to the power 1.1 to ensure that its extreme value index $\gamma$ exceeds unity.

These distributions satisfy all the assumptions of Theorem 2 with $\left(\gamma_{1}, \tau, \beta, \rho\right)=(1,-1,2,-2)$ and $R(x, y)=x+y-\left(x^{2}+y^{2}\right)^{1 / 2}(x, y \geq 0)$ for the Cauchy example, and for the Student $(1 \cdot 1)$ distribution, $\left(\gamma_{1}, \tau, \beta, \rho\right)=(1,-10 / 11,21 / 11,-20 / 11)$ and

$$
R(x, y)=\frac{(1 \cdot 1)^{2} \Gamma(0 \cdot 55)}{\pi^{1 / 2} \Gamma(1 \cdot 05)} \int_{x^{-10 / 11}}^{\infty} \int_{y^{-10 / 11}}^{\infty}\left(v^{2}+w^{2}\right)^{-3 \cdot 1 / 2} d v d w
$$

where $\Gamma$ is the gamma function. Before applying our methodology, the first component is transformed using the logarithm.

To compute our estimator, we need to choose $k_{1}, k_{2}$ and $a_{n}$. We take $k_{1}=\left\lfloor n^{a}\right\rfloor$ and $k_{2}=\left\lfloor n^{b}\right\rfloor$ for $a \in(0, b)$ and $b>0$. From an extensive simulation study, only partly presented in the Supplementary

Material, we observe that our estimation procedure is not overly sensitive to the values of $\left(b, a_{n}\right)$ provided $b \in[0.75,0.85]$ and $a_{n} \in[0.80,0.85]$. According to the conditions of Theorem 2, in particular $a<\min \{-2 \tau(1-b), 2 b-1\}, b$ cannot be too large. We finally chose the values $\left(b, a_{n}\right)=(0 \cdot 75,0 \cdot 85)$ and focus only on the choice of $a$ below. Table 1 gives the absolute value of the empirical bias of $\hat{\theta}_{p} / \theta_{p}$ together with its empirical mean squared error based on $B=500$ samples with different pairs $(n, p)$ in 
Table 1: Absolute value of the empirical bias (empirical mean squared error) of $\hat{\theta}_{p} / \theta_{p}$, multiplied by 100

$\begin{array}{llrrrrr}(n, p) & & (50,0 \cdot 05) & (50,0 \cdot 01) & (50,0 \cdot 001) & (1000,0 \cdot 001) & (1000,0 \cdot 0005) \\ a=0 \cdot 1 & \text { Cauchy } & 35(20) & 2(13) & 11(30) & 2(3) & 1(6) \\ & \text { Student } & 30(16) & 1(15) & 10(29) & 1(3) & 5(7) \\ a=0 \cdot 2 & \text { Cauchy } & 19(8) & 1(12) & 10(23) & 2(3) & 4(5) \\ & \text { Student } & 15(7) & 2(11) & 10(22) & 5(4) & 6(5) \\ a=0 \cdot 3 & \text { Cauchy } & 6(6) & 4(12) & 10(19) & 4(3) & 4(4) \\ & \text { Student } & 4(7) & 5(13) & 8(17) & 5(3) & 5(4) \\ a=0 \cdot 4 & \text { Cauchy } & 7(9) & 10(12) & 14(20) & 3(2) & 3(2) \\ & \text { Student } & 10(9) & 11(14) & 13(19) & 5(2) & 3(2)\end{array}$

Table 2: Absolute value of the empirical bias (empirical mean squared error) of $\hat{\theta}_{p}^{\mathrm{emp}} / \theta_{p}$, multiplied by 100

\begin{tabular}{|c|c|c|}
\hline$(n, p)$ & $(50,0 \cdot 05)$ & $(1000,0 \cdot 001)$ \\
\hline Cauchy & 14 (16) & 7 (7) \\
\hline Studen & 15 (18) & 7 (8) \\
\hline
\end{tabular}

case $\left(b, a_{n}\right)=(0 \cdot 75,0 \cdot 85)$. The size $n=50$ is relatively small, but it illustrates the performance of our estimator in a situation similar to that considered in Section 4. We take $p=0.001$ and 0.0005 as typical values for applications in the financial risk context, but also values such as $p=0.01$ and 0.05 , which are often used in the environmental framework. From Table 1, we can observe that $a$ should not be too small, since it leads to a larger bias and mean squared error, in particular for small $n$. The mean squared error increases when $p$ decreases for a fixed sample size. The choice $a=0.3$ seems to be suitable, whatever the pair $(n, p)$ or the strength of dependence between $Z$ and $Y$.

If $(n, p)=(50,0 \cdot 05)$ and $(1000,0 \cdot 001)$, we have $n p=2.5$ and 1 respectively, allowing us to compare the absolute value of the empirical bias and the empirical mean squared error yielded by our estimator $\hat{\theta}_{p}$ to those obtained with the empirical estimator $\hat{\theta}_{p}^{\text {emp }}=\lfloor n p\rfloor^{-1} \sum_{1}^{n} \log \left(Z_{i}\right) \mathbb{1}_{\left(Y_{i}>Y_{n-\lfloor n p\rfloor n}\right)}$. Table 2 shows the absolute value of the empirical bias of $\hat{\theta}_{p}^{\text {emp }} / \theta_{p}$ together with the mean squared error, based on $B=500$ samples, which have to be compared with the same quantities computed for our estimator in Table 1. The superiority of our estimator is clear, with a bias and mean squared error around 2.5 times smaller than those obtained with the empirical estimator. The relevance of the normal approximation provided by Theorem 2 has been assessed by QQ-plots, which allow us to conclude that Theorem 2 provides an adequate approximation for finite sample sizes.

\section{APpliCATION: TSUNAMI DATA}

We analyze data from the US National Oceanic and Atmospheric Administration on Japanese tsunamis from 1400 to 2011 (http://www.ngdc.noaa.gov/); this dataset is described and studied in Chavez-Demoulin $\&$ Davison (2012). We consider the maximum water height above sea level $Z$, in $\mathrm{cm}$ and the magnitude $Y$, in Richter scale of the earthquake preceding the tsunami. The two variables are upper-tail dependent according to Fig. 7 of Chavez-Demoulin \& Davison (2012). The magnitude of an earthquake being determined from the logarithm of the amplitude of waves recorded by seismographs, working with the logarithm of the maximum water height makes sense. Because the data show trend, we consider a moving window approach with a window size of 50. We use our methodology to estimate the marginal expected shortfall $\theta_{p}$ with $\left(a, b, a_{n}\right)=(0 \cdot 3,0 \cdot 75,0 \cdot 85)$ on each window and for which we get $\hat{\gamma} \geq 1$ when fitting 
a generalized Pareto distribution model on the 50 largest water heights. Figure 1 shows the estimates $\hat{\theta}_{p}$, which suggest that severe earthquakes with a magnitude above the $95 \%$ quantile, in the most recent period, have a smaller impact on the maximum water height, compared to the impact in the oldest period, whereas extremely severe earthquakes with a magnitude above the $99 \%$ quantile have a slightly larger impact in the most recent period. This is due to the fact that, in our dataset, the maximum water height has a decreasing trend, whereas the level of the earthquake magnitude changes less. Furthermore, recent extreme events, such as the 2011 event in Honshu, affect the data, leading to larger confidence intervals.
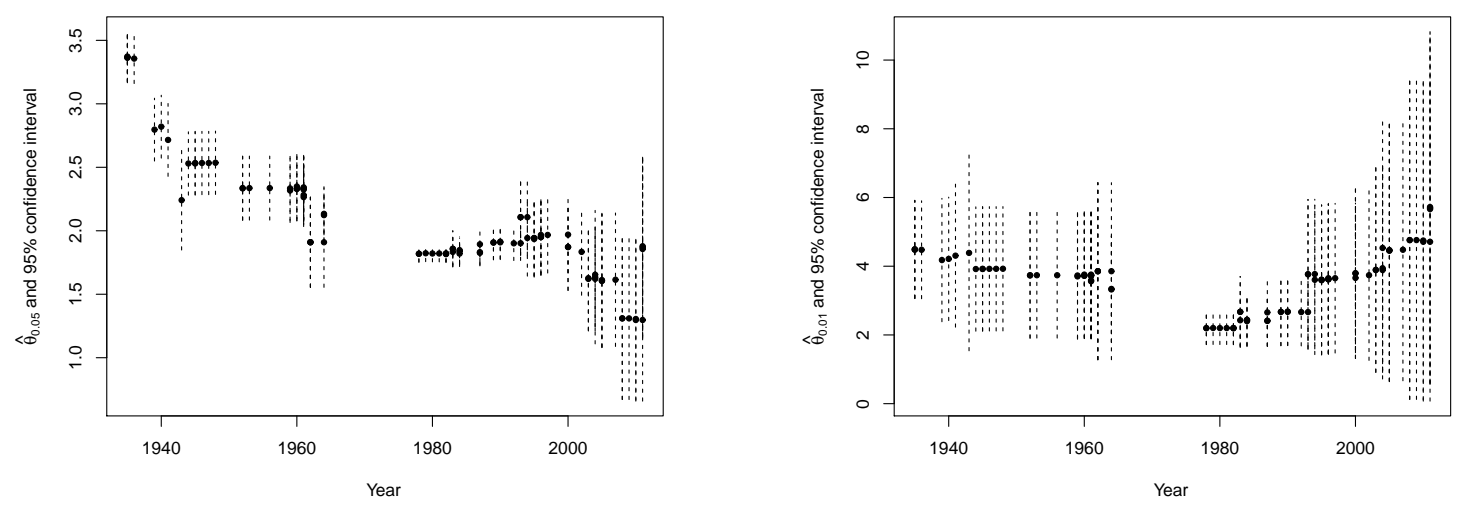

Fig. 1: Tsunami data: estimated values for $\theta_{p}$ and $95 \%$ confidence intervals in moving window with $p=0.05$ (left panel) and $p=0.01$ (right panel).

\section{ACKNOWLEDGEMENT}

Armelle Guillou's work was supported by a research grant from VILLUM FONDEN. We thank the editor, associate editor and the three referees for their helpful comments and Paul Embrechts for his constant encouragement and our discussions on an earlier version.

\section{SUPPLEMENTARY MATERIAL}

Supplementary material available at Biometrika online includes the proofs of Theorems 1 and 2, additional simulations where we investigate the choice of the tuning parameters involved in the estimation procedure, and an application in the operational risk context.

\section{REFERENCES}

Beirlant, J., Goegebeur, Y., Segers, J. \& Teugels, J. (2004). Statistics of Extremes, Theory and Applications. Chichester: Wiley.

CAI, J.-J., EINMAHL, J. H. J., DE HAAN, L. \& Zhou, C. (2015). Estimation of the marginal expected shortfall: the mean when a related variable is extreme. J. R. Statist. Soc. B 77, 417-442.

CAporin, M. \& SAntUCCI DE Magistris, P. (2012). On the evaluation of marginal expected shortfall. Applied Economics Letters 19, 175-179.

Chavez-Demoulin, V. \& DaVison, A. C. (2012). Modelling time series extremes. REVSTAT 10, 109-133.

Chavez-Demoulin, V., Embrechts, P. \& Hofert, M. (2016). An extreme value approach for modeling operational risk losses depending on covariates. J. Risk Insur. 83, 735-776.

HALL, P. (1982). On some simple estimates of an exponent of regular variation. J. R. Statist. Soc. B 44, 37-42.

Hofert, M. \& WUeThrich, M. (2013). Statistical review of nuclear power accidents. Asia-Pacific Journal of Risk and Insurance 7, 1-18. 
RESNICK, S. I. (1997). Heavy tail modeling and teletraffic data: special invited paper (with discussion). Ann. Statist. 25, 1805-1869.

Wheatley, S., Sovacool, B. K. \& Sornette, D. (2016). Reassessing the safety of nuclear power. Energy Research \& Social Science 15, 96-100. 